\title{
SECTOR DE LA ECONOMÍA LABORAL (SEL): UNA VISIÓN COSTARRICENSE
}

\author{
FEDERICO LI BONILLA \\ Escuela de Ciencias de la Administración \\ Universidad Estatal a Distancia, Costa Rica \\ fli@uned.ac.cr \\ GREIBIN VILLEGAS BARAHONA \\ Escuela de Ciencias de la Administración \\ Universidad Estatal a Distancia, Costa Rica \\ gvillegas@uned.ac.cr
}

\section{RESUMEN}

Desde la década de los sesenta en Costa Rica se presenta una propuesta social, económica y políticofilosófica sobre el Sector de la Economía Social; tal iniciativa se basa en el empoderamiento de la propiedad productiva en la mayor cantidad de trabajadores, como un medio para asegurar el futuro económico y, por ende, garantizar de manera sostenible una mejor calidad de vida para el ser humano.

Se corrobora y valida el Sector de la Economía Laboral (SEL) costarricense como un grupo económicosocial, el cual permite el desarrollo y empoderamiento de la propiedad productiva en Costa Rica. EI SEL es una propuesta política-económica-social, la cual pretende vincular empresas y asociaciones sociolaborales, juntas de pensiones, el Banco Populary Desarrollo Comunal, cooperativas, asociaciones solidaristas, las asociaciones administradoras de sistemas de acueductos y alcantarillados comunales (ASADAS); así como toda aquella organización de carácter administrativo-democrática, cuyo fin sea el bienestar del ser humano, bajo una ley marco. En este documento se propone abrir el debate científco-político de una propuesta alterna a la Economía Social, y apoyar la creación de una ley general marco que articule, fomente, integre y consolide el Sector de la Economía Laboral en Costa Rica.

PALABRAS CLAVE: ECONOMÍA LABORAL, EMPODERAMIENTO, SINDICALISMO, COOPERATIVISMO, SOLIDARISMO, ECONOMÍA SOCIAL, EMPRESAS SOCIOLABORALES, CONGLOMERADOS O CLÚSTERS.

\begin{abstract}
Since the 1960's, Costa Rica has adopted a politico-philosophical, social and economic model in relation to its socialeconomic sector; such proposal is based on the empowerment of the productive property among the largest possible quantity of workers, as a means to ensure their economic future and, therefore, the sustainability of a better quality of life for the people. This essay validates the Costa Rican labor economic sector (SEL) as a socio-economic group that allows the development and empowerment of the country's productive property. SEL is a socio-political and economic proposal intended to link enterprises, socio-labor associations, retirement associations, the Banco Populary Desarrollo Comunal, cooperatives, solidarity associations, community sewage and water administration systems associations (ASADAS), and almost every other administrative democratic organization which aim is the general wellbeing into the same legal framework. This essay opens a political-scientific debate in relation to a proposal alternative to social economy and supports the establishment of a general legal framework to articulate, stimulate, integrate and consolidate Costa Rica's labor economic sector.
\end{abstract}

KEYWORDS: LABOR ECONOMICS, EMPOWERMENT, UNIONS, COOPERATIVES, SOLIDARISM, SOCIAL ECONOMY, LABOR PARTNER COMPANIES, CLUSTERS.

\section{INTRODUCCIÓN}

El presente artículo es resultado de una investigación en el campo del sector de economía laboral. Se expone a la validación o rechazo de los costarricenses sobre este sector, por el hecho de 
ser una propuesta político-filosófico-económica originada de la más genuina convicción de Francisco Morales Hernández, en la cual propone que Costa Rica debe tomar otro rumbo. Por ejemplo, cambiar las ambiciones, aspirar a participar de la verdadera creación de riqueza en el mundo actual a través del conocimiento, la innovación y el emprendimiento. De manera que, Francisco Morales propone la creación de las dos E: empresarios en vez de empleados. A partir de ello, sugiere que se deben crear nuevas formas de valor generadoras de puestos de trabajo de alta calidad, los cuales jerarquicen y aprovechen la educación y la cultura tan distintiva de Costa Rica como país. La propuesta sobre economía laboral es una de las supremas expresiones de creación de valor a partir de la creatividad humana.

\section{Antecedentes del Sector de la Economía Laboral}

El SEL es una propuesta ambiciosa e innovadora que don Francisco Morales, exministro de Trabajo, viene impulsando desde 1968, la cual se fundamenta en la filosofía política y social, de la doctrina política de la social democracia. Es así como en un cuaderno del Centro de Estudios Democráticos de América Latina (CEDAL, 1978) el exvicepresidente de la República, ex Oficial Mayor del Ministerio de Trabajo y Seguridad Social, exmiembro de la Junta de Gobierno de 1948, diputado nacional en dos ocasiones, catedrático de la Universidad de Costa Rica, primer abogado laboral, además de Trabajador Social (licenciado en ambas carreras), Armando Arauz Aguilar (Arauz, Morales, Rojas, Lewinsky y Livchen, 1978) dice:

... Costa Rica tiene la oportunidad, a mi juicio único en América Latina, de crear sus propias soluciones, de estructurar un esquema propio, nuevo e imaginativo para seguir siendo un país libre políticamente, económicamente equilibrado y socialmente justo. (...) Ese camino, pienso yo, está claramente delineado con la creación de un sector de la economía laboral (SEL) dentro del esquema actual de la vida nacional (pp. 21-22).

Por su parte, don Rafael Rojas Jiménez, exministro de Cooperativas, exministro de Trabajo y Seguridad Social, exdiputado (Arauz et al., 1978), opinó hace 35 años:

... sobre la idea de establecer un Sector de la Economía Laboral en nuestro país, responde a la necesidad de encontrar soluciones viables para enfrentar muchos de los problemas que, a pesar de los esfuerzos hechos, aún persisten en nuestra sociedad.

En este contexto, los propósitos del SEL son de contribuir a solucionar problemas de empleo y distribución del ingreso, a través de la participación de los trabajadores... (p. 39).

De esta forma, el Gobierno del Estado de Israel envía en una misión de dos semanas, del 29 de junio al 15 de julio de 1977, a un emisario para realizar una asesoría sobre el SEL. Esta misión recae en manos de Akiva Lewinsky, consultor de la Organización de Estados Americanos y miembro, como directivo, de más de veinte organizaciones sociolaborales en Israel y en el resto del mundo. Su objetivo fue determinar las posibilidades para la creación y desarrollo del SEL, con especial énfasis en los instrumentos de financiamiento y otros necesarios para tal proyecto. A partir de su informe rendido se rescata, entre otras observaciones positivas, lo siguiente (Arauz et al., 1978, p. 62):

1. La idea de un Sector de Economía Laboral es producto del señor Francisco Morales Hernández. (...)

2. La OIT recibió esta idea con beneplácito considerándola como una respuesta importante para la solución de los problemas de desempleo, del desarrollo económico y social y del fortalecimien- 
to del sistema democrático costarricense, constituyendo un paso adelante hacia la democracia económica (...)

3. La idea del SEL es una contribución original de Costa Rica con características propias y definidamente costarricense, que coinciden y responden en un todo con los principios expresados en los últimos diez años en la Conferencia de Ministros de Trabajo de América... (p. 62).

Para noviembre de 1977, la Organización Internacional del Trabajo (OIT) designó por 22 días, a solicitud del Gobierno de Costa Rica, un técnico para el análisis de la propuesta del SEL; siendo este el señor René Livchen, graduado en Ciencias Económicas de la Universidad de Ginebra, Suiza.

En su informe, entre otras cosas, hace referencia a que el Ministerio de Trabajo quiere implantar el SEL en Costa Rica, ello con la finalidad de mejorar la distribución de ingresos y bajar la brecha socioeconómica del país. En el marco de referencia del informe realizado, Livchen acota lo siguiente (Arauz et al., 1978):

5. Existen numerosas resoluciones internacionales y estudios que han recalcado la importancia de considerar -en una política de desarrollo con objetivos de empleo, distribución de ingresos y satisfacción de las necesidades básicas de la población- las medidas nacionales tendientes a transformar la estructura de producción y de propiedad, con la finalidad de asegurar a largo plazo mejores condiciones de vida y de empleo a la población (p. 94).

Después de realizar un estudio exhaustivo sobre el SEL, Livchen, en lo referente a marco de referencia, justificación, antecedentes, estado actual del proyecto, objetivos del SEL, principios básicos, instituciones principales del SEL, finan- ciamiento propuesto, resultados esperados, así como los programas sectoriales colaterales; de analizar los acuerdos y desacuerdos para ese momento (1977), así como las actitudes empresariales y sindicales, llega a la conclusión de la viabilidad y necesidad de implementar el Sector de Economía Laboral, en el cual reconoce la necesidad formativa de algunas personas en el campo de la economía laboral. Por ello, en el punto 74 de su informe (Arauz et al., 1978, p. 118), Livchen concluye:

74. La OIT estaría dispuesta a ayudar en la búsqueda de fuentes de financiación para la cooperación técnica que requiere la puesta en marcha del SEL. Para ello, se podría pensar en el PNUD y en la ayuda multilateral de países como Holanda, Suecia, Canadá, Bélgica, Alemania, etc., que por el carácter temático y de programa piloto que tendría el SEL en Costa Rica estarían eventualmente interesadas...

Por su parte, el autor de esta idea filosófica y política, Francisco Morales (2008), plantea sus inquietudes y propuestas del valor ético del trabajo. Afirma que la verdadera democracia se da cuando existen recursos económicos sumados al empoderamiento de la propiedad productiva (bienes o servicios) en la mayor cantidad de los trabajadores, lo cual permita tener un mejor ingreso económico. De manera que se promuevan mejores condiciones de vida democrática, sobre la base de una mayor participación de las personas en la producción y distribución de la riqueza nacional mediante la creación de un Sector de Economía Laboral.

Democratizar la democracia es una aspiración permanente de nuestros pueblos. Siempre habrá espacios nuevos que cubrir en procura de contar con mejores condiciones para el desarrollo del ser humano. 
Los resultados que se analizan validan la aceptación y comprueban la percepción del pueblo costarricense sobre las propuestas de la economía laboral, con ello se visibilizan las necesidades y aspiraciones de los pueblos. Conocer sus necesidades y su pensamiento ha reflejado en esta investigación que la economía laboral es pertinente para lograr una mejor y mayor clase media en Costa Rica, crear mejores y más empleos, contribuir a optimizar la calidad de vida del costarricense, logrando de esta manera "un país de propietarios y menos proletarios".

Para elaborar esta investigación se utilizó, como apoyo y herramienta, la plataforma Moodle, así como el acceso a la plataforma de videoconferencias de la Universidad Estatal a Distancia (UNED). Las encuestas fueron realizadas por, aproximadamente, 800 estudiantes de los cursos de estadística. La UNED cuenta con 37 centros y subcentros universitarios distribuidos estratégicamente en todo el país. Por lo tanto, los resultados de esta investigación son una muestra homogénea de las poblaciones costarricenses.

En este estudio se someten a validación los conceptos propuestos por Morales, a partir de los cuales, entre otros temas, se consulta sobre el valor ético del trabajo, de cómo se podría invertir de manera alternativa los fondos de las cooperativas, asociaciones solidaristas, pensiones complementarias, pensiones de la Caja Costarricense del Seguro Social, Junta de Pensiones y Jubilaciones del Magisterio, el Banco Popular y Desarrollo Comunal (banco de los trabajadores), entre otras agrupaciones de índole laboral.

También, se tiene la aceptación de que los recursos de los trabajadores deberían invertirse en infraestructura del país, tales como aeropuertos, puertos, carreteras, ferrocarriles, entre otras obras públicas. Esto le permitiría tener mejores excedentes a los trabajadores, como asociados a cooperativas, asociaciones solidaristas; y a los pensionados, mejores cuotas.

\section{¿Qué se entiende por Sector de la Economía Laboral?}

Sintetizando lo propuesto por el autor intelectual sobre el SEL y los estudios realizados por consultores internacionales, así como políticos costarricenses, se infiere que el Sector de la Economía Laboral se basa en el empoderamiento de la propiedad productiva en la mayor cantidad de los trabajadores, como un medio que asegura el futuro económico y, por ende, da sostenibilidad para una mejor calidad de vida del ser humano.

Además, respeta los términos jurídicos que gozan las diferentes formas en las cuales se agrupan, para su representación, los trabajadores, así como el autoempleo, conferido según la ley; pudiendo ser cooperativas, asociaciones solidaristas, juntas de pensiones, sindicatos, municipalidades, colegios profesionales, sociedades anónimas laborales (SALES), juntas comunales, asociaciones de campesinos, etc. Ello, sin menoscabo de las empresas familiares y microempresas. No obstante, sin dejar de exaltar el valor ético del trabajo, la democratización económica y, sobre todo, la democracia política.

\section{Diseño metodológico de la investigación}

El presente artículo es una investigación mixta, comprende investigación de campo (representada primordialmente por las encuestas); además, la triangulación conlleva entrevistas abiertas a diferentes personeros quienes han propuesto el Sector de Economía Laboral, por ejemplo: los exministros de Trabajo y Seguridad Social de Costa Rica, Francisco Morales y Rafael Ángel Rojas. Así como la consulta a las diferentes organizaciones gremiales asociativas financieras de Costa Rica. Finalmente, se llevó a cabo una revisión exhaustiva de lo escrito hasta el momento y de carácter transversal. 


\section{Población de estudio según datos del censo 2011}

La población de estudio para esta investigación fueron todos los costarricenses o residentes en el territorio nacional con edad entre 17 y 60 años, y el período fue de noviembre de 2011 a febrero de 2012. Para el Censo Nacional de Población, se obtuvo 3466654 personas en Costa Rica, la población de interés es de 2655320 personas; de las cuales se tiene un total de 1582530 ocupados y 1072790 no ocupados. Entre los no ocupados se incluyeron los que buscan trabajo, los que buscan trabajo por primera vez, los inactivos, pensionados o jubilados, los inactivos que viven de rentas o alquileres, los que estudian, y quienes se dedican a oficios domésticos e inactivos. En las siguientes tablas se muestra en detalle la población de estudio. Para referencias de estimaciones globales el total es de 2655320 personas.

\section{Diseño estadístico de la muestra}

La muestra utilizada en esta investigación fue de 3394 personas, con la cual se realizan estimaciones de los parámetros poblacionales, con una confianza del 95\% y un error máximo permitido de 1,68\%; es decir, toda diferencia superior al 1,68\%, entre las proporciones observadas, es evidencia de diferencia significativa.

\section{Muestreo estadístico y ponderación de la muestra}

El diseño de muestreo en esta investigación fue por cuota probabilística, de tal manera, se controló el informante por género, edad y si trabaja o no. Por lo general, las muestras diseñadas por cuota tienen sesgos de selección muy fuertes, pues no son una muestra aleatoria pura; por ello, se debe tener el cuidado estadístico de ponde-

TABLA 1

\section{POBLACIÓN DE COSTA RICA SEGÚN EL CENSO NACIONAL DE POBLACIÓN 2011, SEGÚN EDAD POR CONDICIÓN DE OCUPACIÓN}

\begin{tabular}{lcccc}
\multicolumn{1}{c}{ CONDICION DE OCUPACIÓN } & EDAD & & TOTAL \\
\multicolumn{1}{c}{ Ocupados } & 12 a 16 & 17 a 60 & 61 a 112 & 1674275 \\
Desocupado, buscó trabajo y había trabajado antes & 12521 & 1582530 & 79224 & 50981 \\
Desocupado, buscó trabajo primera vez & 920 & 48777 & 1284 & 8108 \\
Inactivo pensionado o jubilado & 621 & 7419 & 68 & 185245 \\
Inactivo vive de rentas o alquileres & 164 & 36665 & 148416 & 8916 \\
Inactivo solo estudia & 183 & 4991 & 3742 & 588299 \\
Inactivo se dedica a oficios domésticos & 329178 & 258419 & 702 & 680354 \\
Inactivo otra situación & 16,074 & 545566 & 118714 & 270476 \\
\hline TOTAL & 33929 & 170953 & 65594 & 3466654 \\
\hline
\end{tabular}

Fuente: Instituto Nacional de Estadística y Censo, 2011. 
rar los resultados según la estructura de la población objeto de estudio. Cuando la muestra por cuota se diseña proporcionalmente con las características de estructura de la población y se logra cumplir a cabalidad con esta, entonces no se requiere de dicha ponderación dado que la misma muestra reproduce esas estructuras, pero normalmente, por las condiciones de encuesta siempre se observan diferencias entre lo diseñado y lo finalmente obtenido en el estudio. Por ello, es necesario el análisis de la estructura de la muestra en comparación con la estructura de la población para evitar estimaciones sesgadas.

En este caso, las variables con las cuales se ponderó la muestra fueron: escolaridad, sexo, edad, ocupación, provincia y nivel socioeconómico.

Por ejemplo, en la población de interés, el 21,0\% de los hombres está desocupado, y en el caso de las mujeres, el 58,6\%; mientras que en la muestra se indica que el 11,6\% de los hombres es no ocupado y para las mujeres dicho porcentaje es 23,7\%; eso significa que la muestra no es representativa por condición de ocupación y género, según se aprecia en la tabla 2. Entonces, al encontrar esas diferencias se procedió a la ponderación de la muestra, según el siguiente formato de ponderación para esas variables.

TABLA 2

\section{OCUPACIÓN POR GÉNERO}

\begin{tabular}{|c|c|c|}
\hline \multirow{2}{*}{ GÉNERO } & \multicolumn{2}{|c|}{ OCUPADO O NO OCUPADO } \\
\hline HOMBRE & NO OCUPADO & OCUPADO \\
\hline MUJER & 1,81 & 0,89 \\
\hline
\end{tabular}

Fuente: Elaboración propia.

Al ponderar la muestra por estos factores, se logra que esta se comporte, aproximadamente, a la composición real de la población, según se puede ver en la tabla 3.
TABLA 3

\section{PROPORCIÓN DE LA POBLACIÓN Y LA MUESTRA OBTENIDA SEGÚN GÉNERO REFERENTE A LA OCUPACIÓN}

\begin{tabular}{|c|c|c|c|c|c|c|}
\hline \multirow{2}{*}{ GÉNERO } & \multicolumn{2}{|c|}{ POBLACIÓN CENSO } & \multicolumn{2}{|c|}{$\begin{array}{l}\text { MUESTRA NO } \\
\text { PONDERADA }\end{array}$} & \multicolumn{2}{|c|}{$\begin{array}{c}\text { MUESTRA } \\
\text { PONDERADA }\end{array}$} \\
\hline & Ocupado & $\begin{array}{c}\text { No } \\
\text { ocupado }\end{array}$ & Ocupado & $\begin{array}{c}\text { No } \\
\text { ocupado }\end{array}$ & Ocupado & $\begin{array}{c}\text { No } \\
\text { ocupado }\end{array}$ \\
\hline HOMBRE & $79,0 \%$ & $21,0 \%$ & $88,4 \%$ & $11,6 \%$ & $78,3 \%$ & $21,7 \%$ \\
\hline MUJER & $41,4 \%$ & $58,6 \%$ & $76,3 \%$ & $23,7 \%$ & $43,3 \%$ & $56,7 \%$ \\
\hline TOTAL & $59,6 \%$ & $40,4 \%$ & $84,2 \%$ & $15,8 \%$ & $60,8 \%$ & $39,2 \%$ \\
\hline
\end{tabular}

Fuente: Elaboración propia.

Ahora bien, lo mismo ocurre con la estructura de escolaridad. La selección de la muestra estaba desproporcionada por personas de mayor escolaridad, universitarios en su mayoría, mientras que la participación de informantes de primaria no estaba siendo bien representada; no obstante, al ponderar por la estructura poblacional la muestra final reproduce esta estructura.

\section{TABLA 4}

\section{PONDERACIÓN DE LA MUESTRA SEGÚN POBLACIÓN REFERENTE A LA ESCOLARIDAD}

\begin{tabular}{|l|c|c|c|}
\hline \multicolumn{1}{|c|}{ ESCOLARIDAD } & $\begin{array}{c}\text { POBLACIÓN } \\
\text { CENSO }\end{array}$ & $\begin{array}{c}\text { MUESTRA SIN } \\
\text { PONDERAR }\end{array}$ & $\begin{array}{c}\text { MUESTRA } \\
\text { PONDERADA }\end{array}$ \\
\hline PRIMARIA & $31,5 \%$ & $9,8 \%$ & $32,0 \%$ \\
\hline SECUNDARIA & $29,9 \%$ & $25,5 \%$ & $39,4 \%$ \\
\hline TÉCNICO & $6,8 \%$ & $8,8 \%$ & $7,2 \%$ \\
\hline $\begin{array}{l}\text { UNIVERSIDAD } \\
\text { INCOMPLETA }\end{array}$ & $11,2 \%$ & $16,5 \%$ & $11,3 \%$ \\
\hline $\begin{array}{l}\text { UNIVERSIDAD } \\
\text { COMPLETA }\end{array}$ & $7,0 \%$ & $34,1 \%$ & $6,8 \%$ \\
\hline POSTGRADO & $3,6 \%$ & $5,3 \%$ & $3,3 \%$ \\
\hline TOTAL & $100,0 \%$ & $100,0 \%$ & $100,0 \%$ \\
\hline
\end{tabular}

Fuente: Elaboración propia. 
Una de las variables utilizadas más importantes para generalizar los resultados, a nivel de todo el país, es la provincia de residencia de los informantes; evidentemente la muestra quedó desproporcionada por provincia, dándole mayor peso a los informantes de San José, tal como se puede observar en la tabla 5. No obstante, al ponderar la muestra según la estructura de provincia de residencia de la población de estudio, se logra darle el peso correspondiente a las respuestas de cada informante según la provincia donde reside.

TABLA 5

\section{PONDERACIÓN DE LA MUESTRA POR PROVINCIA SEGÚN CENSO 2011}

\begin{tabular}{|l|c|c|c|}
\hline \multicolumn{1}{|c|}{ PROVINCIA } & $\begin{array}{c}\text { POBLACIÓN } \\
\text { CENSO }\end{array}$ & $\begin{array}{c}\text { MUESTRA SIN } \\
\text { PONDERAR }\end{array}$ & $\begin{array}{c}\text { MUESTRA } \\
\text { PONDERADA }\end{array}$ \\
\hline SAN JOSÉ & $33,3 \%$ & $41,5 \%$ & $33,0 \%$ \\
\hline ALAJUELA & $19,6 \%$ & $18,6 \%$ & $19,6 \%$ \\
\hline CARTAGO & $11,6 \%$ & $14,6 \%$ & $12,0 \%$ \\
\hline HEREDIA & $10,4 \%$ & $9,9 \%$ & $10,4 \%$ \\
\hline GUANACASTE & $7,4 \%$ & $4,4 \%$ & $7,4 \%$ \\
\hline PUNTARENAS & $9,1 \%$ & $6,2 \%$ & $9,1 \%$ \\
\hline LIMÓN & $8,5 \%$ & $4,7 \%$ & $8,5 \%$ \\
\hline TOTAL & $100,0 \%$ & $100,0 \%$ & $100,0 \%$ \\
\hline
\end{tabular}

Fuente: Elaboración propia.

Finalmente, al aplicar las correcciones anteriores por medio de las ponderaciones, en esta investigación se garantiza que las estimaciones con los resultados obtenidos son confiables, fidedignas y sin sesgo de selección dadas las diferencias entre la composición de la muestra y la población de interés.

\section{Diseño del instrumento recopilación de datos}

El diseño del instrumento utilizado en esta investigación fue un cuestionario estructurado autoadministrado por internet, consta de dos secciones; la primera parte, compuesta por ocho características de perfil y control demográfico, estas características son: edad, sexo, residencia (provincia), ocupación, escolaridad, nivel socioeconómico, actividad principal y posición en el empleo; la segunda parte, incluye 33 variables sustantivas de investigación sobre el SEL, con las cuales el informante brinda su grado de acuerdo o desacuerdo con la afirmación. Para ello se utilizó una escala de Likert de cinco puntos, desde muy en desacuerdo hasta muy de acuerdo.

La idea era que las personas expresaran su grado de acuerdo o desacuerdo a cada una de las afirmaciones planteadas. Debido a esto, fueron construidas tomando como referencia los conceptos propios del sector de la economía laboral, tales como económicos, filosóficos, políticos y sociales.

\section{Método de recopilación de datos}

Para la recopilación de datos se utilizaron entrevistadores de todo el país, los estudiantes del curso Estadística I, de la Universidad Estatal a Distancia, colaboraron con la búsqueda de informantes. Cada uno ubicó a cuatro personas, de las cuales tres fueran hombres trabajadores, uno con edad de 40 a 60 años, otro de 30 a 39 años, y un tercero de 20 a 29 años. El cuarto informante debía ser una mujer de 20 a 60 años, trabajadora. Por otro lado, el estudiante también participaba de la investigación como informante.

El estudiante mostró dos videos a los informantes, luego visitaron un sitio en internet para contestar el cuestionario. 
Una vez identificados los informantes, el estudiante les mostraba dos videos con el propósito de que conocieran los conceptos del Sector de la Economía Laboral. Luego de observarlos, accedían al cuestionario publicado en internet. Con esta metodología se logró que los informantes, al responder cada una de las 33 afirmaciones, tuvieran criterio sobre sus respuestas.

\section{Metodología utilizada en el análisis estadístico}

Para realizar este estudio, se empleó la técnica de la segmentación de la población costarricense. Ello, en función de la actitud y percepción de los entrevistados sobre los conceptos del Sector de la Economía Laboral.

\section{Análisis de confiabilidad}

Para este estudio se realizó un análisis de confiabilidad con las 33 afirmaciones, las cuales brindaron un coeficiente de Cronbach de 0,901. Este resultado permite asegurar que las afirmaciones fueron construidas de manera correcta para continuar con la interpretación de factores.

\section{Análisis de factores}

Seguidamente, se procede al análisis de factores con el propósito de identificar variables subyacentes, o bien, factores que expliquen la configuración de correlaciones dentro de las 33 variables observadas.

Este análisis permite construir seis factores, los cuales explican el $75,1 \%$ de la variabilidad total de las 33 características, con una medida de adecuación muestral de Kaiser-Meyer-Olkin de 0,95 y un valor chi-cuadrado de 38043,0 correspondiente a la prueba de esfericidad de Bartlett con un nivel de significancia de 0,000
Con estos factores obtenidos se procedió a efectuar el primer análisis de aglomeración.

\section{FIGURA 1 \\ EL CAMINO RECORRIDO PARA LA SEGMENTACIÓN}

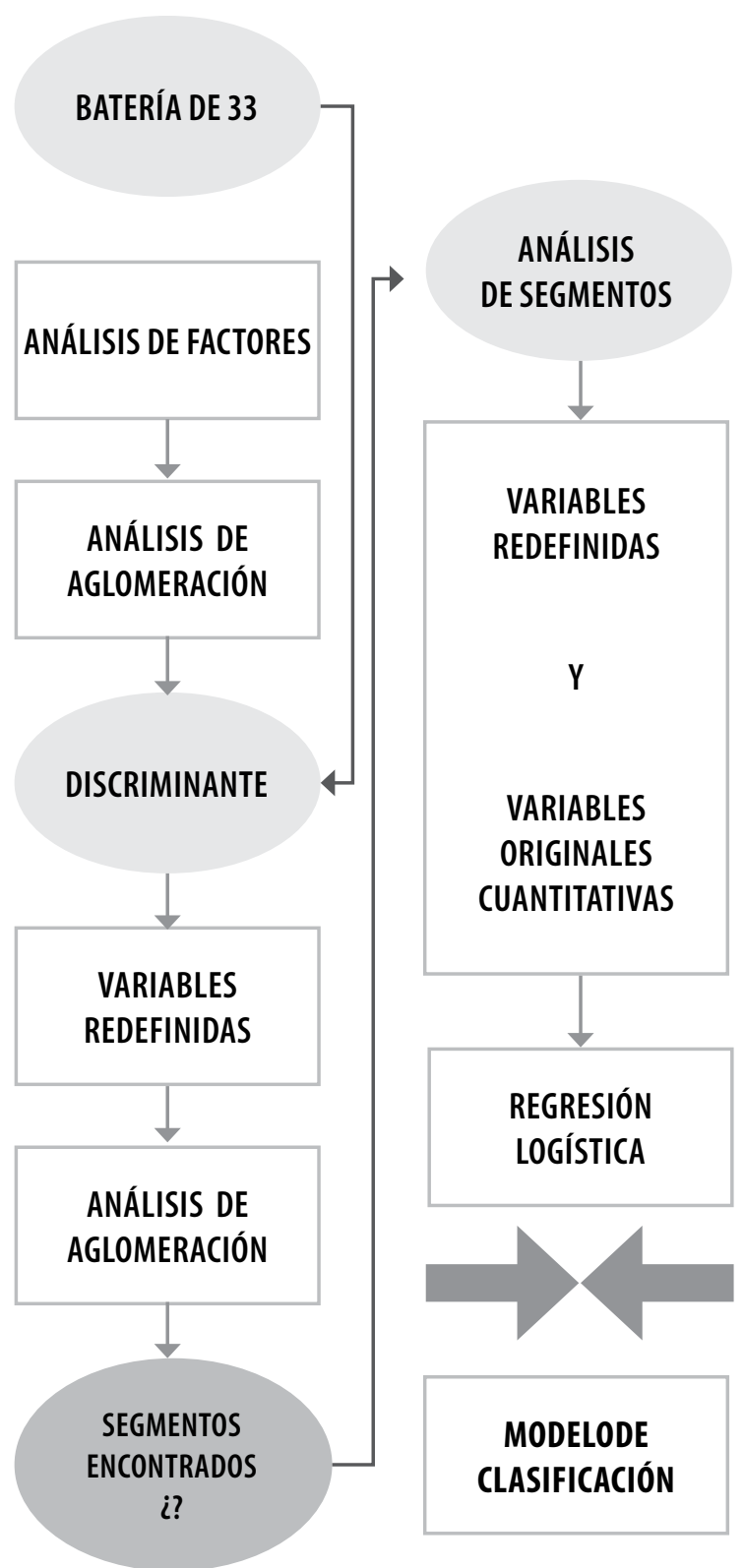

Fuente: Elaboración propia. 


\section{Análisis de aglomeración (Cluster Analysis)}

Esta técnica de análisis multivariable persigue la formación de grupos (clústeres) con los sujetos. De manera que, por una parte los conjuntos reúnan los individuos más homogéneos, y por otra parte, estos sean lo más heterogéneo posible entre sí. La idea es segmentar a la población costarricense en función de los factores obtenidos, los cuales representan dimensiones aproximadas a la actitud de esta población hacia el Sector de la Economía Laboral.

Una vez obtenidos los primeros clusters, se procede a un análisis discriminante con el fin de mejorar la pertenencia de los individuos en cada segmento. Esto debido a que, normalmente, el primer resultado de la aglomeración no es tan eficiente, pues es producto de un procedimiento matemático (distancia euclídea) sin tener tanta influencia el método estadístico (maximización de la variancia) en su conformación.

\section{Análisis discriminante}

El análisis discriminante es una técnica para explicar la pertenencia de un elemento a un grupo dado, en función de las variables independientes existentes; también, permite predecir a qué grupo pertenecerá un individuo o elemento nuevo del cual se desconoce el valor de una variable dependiente. En esta investigación se utilizó la técnica discriminante para mejorar la construcción de los clusters encontrados en la fase anterior; esta depuración se realizó en tres ocasiones, pasando de un $77,5 \%$ de buena clasificación en la primera corrida del análisis discriminante, a un 96,9\% de buena clasificación en la tercera corrida de este modelo. Por este motivo, los individuos ubicados en cada uno de los segmentos son altamente homogéneos entre sí, es decir, tienden a tener una actitud y percepción muy similar sobre los conceptos del Sector de la Economía Laboral.

\section{Resultados de la investigación sobre la aceptación y percepción del SEL}

Para llegar a la inferencia de los resultados, es necesario identificar los diferentes factores, reduciéndolos al máximo con el objetivo de comprender el criterio de las personas entrevistadas en todo el territorio de Costa Rica.

\section{Derivaciones del análisis}

Para el análisis de factores se utilizaron 33 variables relacionadas con los conceptos del Sector de la Economía Laboral. A partir de ello, se logró reducir, de manera considerable, a seis factores que explican el $75 \%$ de la variabilidad total. Estos son:

Factor 1: (Sector de Economía Laboral). El empoderamiento de la clase media y el mejoramiento del bienestar de los costarricenses.

Factor 2: (Empresa comunitaria). La empresa comunitaria y la gestión municipal para el desarrollo del país.

Factor 3: (Inversión gremial). La inversión gremial en la infraestructura nacional para el desarrollo del país, como un medio democratizador de la propiedad productiva para el bienestar social.

Factor 4: (Trabajo). El fortalecimiento del salario y el valor ético del trabajo humano sobre la empresa y el mercado.

Factor 5: (Democracia económica). El SEL se preocupa por el bienestar de las personas, considera que el salario no contribuye a la creación de un capital, pero produce democracia económica por medio del empoderamiento de la propiedad productiva en los trabajadores (asalariados y por cuenta propia).

Factor 6: (Reivindicación laboral). Costa Rica se encuentra en una etapa creciente con respecto a hace 40 años. 
Con el método jerárquico de Ward, fue posible construir los cuatro conglomerados de costarricenses; este consiste en integrar los distintos individuos en clusters; de tal manera, se mide a través de la suma total de los cuadrados de las desviaciones entre cada punto (individuo) y la media del cluster en el cual se integra. La idea es minimizar la varianza dentro de los clusters para hacerlos más homogéneos y maximizarla entre ellos para diferenciarlos más.

Para que el proceso de clusterización resulte óptimo, en el sentido de que los grupos formados no distorsionen los datos originales, este método de Ward es uno de los más utilizados en la práctica; posee casi todas las ventajas del método de la media y suele discriminar más cuando se determinan los niveles de agrupación.

\section{Clusters encontrados}

El primer cluster, con un tamaño del $24,1 \%$ de la población, por sus características se denomina "Activistas del SEL"; el segundo grupo, Ilamado "Seguidores activos del SEL", representa una cuarta parte de la población costarricense; el tercero corresponde a quienes, por sus características, se les ha denominado "Seguidores pasivos del SEL"; y, finalmente, el cuarto grupo, que es el más pequeño con un 9,2\%, se les ha llamado "Pasivos conscientes del SEL".

La composición interna de estos grupos es altamente homogénea, de tal manera que al caracterizarlos de forma cualitativa es posible determinar un perfil según su actitud y percepción sobre los conceptos del Sector de la Economía Laboral.

\section{Cluster 1: ACTIVISTAS DEL SEL}

Este conglomerado tiene una representación del 24,1\% de la población costarricense. Presentan una afinidad muy alta con el constructo SEL, "Clase media más empoderada para mejorar el bienestar de los costarricenses", al igual que una gran aceptación e interés en que haya una inversión gremial en la infraestructura del país. Están muy de acuerdo con que la economía laboral fortalece el trabajo y da valor ético al esfuerzo

\section{SEGMENTACIÓN SEGÚN EL MÉTODO WARD}

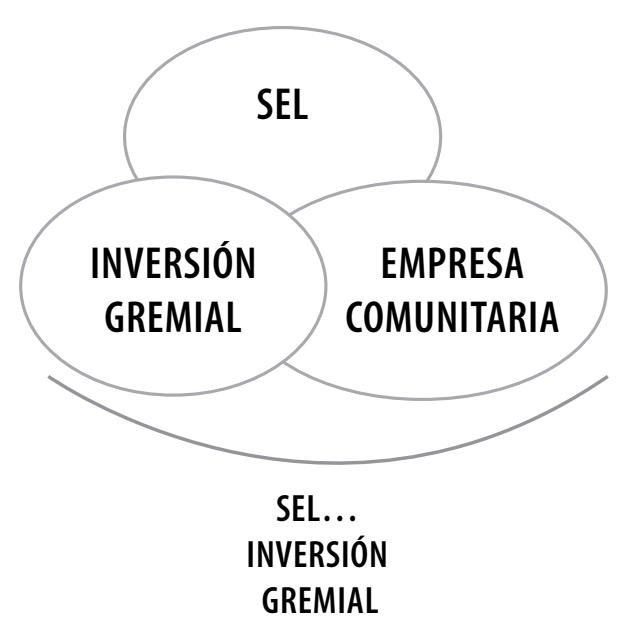

Fuente: Elaboración propia
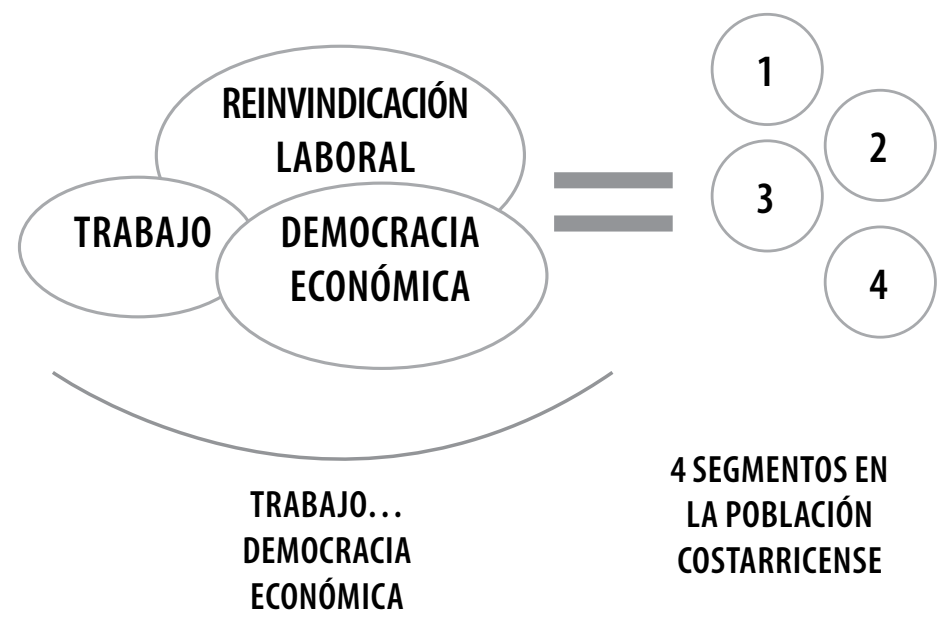

4 SEGMENTOS EN

LA POBLACIÓN

COSTARRICENSE 
humano sobre la empresa y mercado. También, expresan de manera significativa que no existe una democracia económica en Costa Rica, así como la ausencia en la actualidad de la reivindicación laboral. Finalmente, no están convencidos de que el sindicalismo esté velando por los trabajadores.

\section{Cluster 2: SEGUIDORES ACTIVOS DEL SEL}

Este conglomerado está compuesto por el 28,2\% de la población costarricense. Por un lado, tienden a expresar fuertemente que no existe una democracia económica, ni un empoderamiento de la clase trabajadora; por otro lado, afirman que la empresa comunitaria y la municipal son muy importantes. Manifiestan la importancia de la inversión gremial en la infraestructura del país. Perciben, altamente, que el trabajo no fortalece el salario ni dignifica al trabajador.

\section{Cluster 3: SEGUIDORES (PASIVOS) DEL SEL}

Esta agrupación de personas quienes representan el 32,2\% de los costarricenses considera que no existe una democracia económica y el empoderamiento de la clase trabajadora. No consideran que la empresa comunitaria y la empresa municipal contribuyan al desarrollo del país. Están ligeramente de acuerdo con la inversión gremial en el desarrollo de infraestructura del país. Están muy de acuerdo en el fortalecimiento del salario y el reconocimiento del valor ético del trabajo; así como en la democratización de la economía laboral; que el salario contribuye a crear capital; y la existencia de propuestas de desarrollo para el país, las cuales involucren e incluyan a los trabajadores. Por último, afirman que el país se encuentra algo mejor que hace 40 años. En relación con el sindicalismo, destacan su baja influencia en la defensa de los trabajadores.

\section{Cluster 4: PASIVOS CONSCIENTES DEL SEL}

En este conglomerado, formado por el 15,4\% de los encuestados, se tiene que están de acuerdo en que la economía laboral es pertinente para volver a tener una mayor clase media. Además, consideran la propuesta del SEL sobre el empoderamiento de la sociedad trabajadora costarricense en la Organización y Participación Popular, como una forma de contribuir a mejorar el bienestar de los costarricenses y la creación de nuevos empleos. En proporción, a mayor organización del pueblo, mejor reconocimiento a las propuestas de la ciudadanía. Asimismo, para este grupo es muy importante la empresa comunitaria y la municipal para el desarrollo del país. Por un lado, no consideran de manera muy significativa la importancia de la inversión gremial en el desarrollo de infraestructura del país. Por otro lado, creen que el Sector de la Economía Laboral es un mecanismo para fortalecer el salario y propone el valor ético del trabajo humano sobre la empresa y el mercado. En conclusión, aseveran que los diferentes modelos económicos tales como el comunismo y el capitalismo no se preocupan por el bienestar de las personas; no consideran el salario normal de un costarricense como una ayuda a crear un capital; no creen que exista democracia económica; no hay una propuesta de desarrollo para un país, la cual involucre e incluya a los trabajadores como dueños, propietarios o inversionistas de negocios rentables como carreteras, aeropuertos, puertos o bancos; y por último, que hoy se está peor en comparación con hace 40 años, y que el sindicalismo apenas defiende los derechos de los trabajadores.

En síntesis, a partir de los clusteres aislados en esta investigación, como se puede corroborar en las páginas anteriores, es posible inferir, con una alta fiabilidad, que un 79,7\% (como se puede apreciar en el gráfico 1) de las personas entrevistadas, están de acuerdo con los diferentes planteamientos propuestos en el Sector de la 
Economía Laboral (SEL) como la ética del trabajo y el empoderamiento de la propiedad productiva en manos de la mayor cantidad de los trabajadores de Costa Rica. Así como invertir los fondos de las organizaciones laborales en diferentes proyectos de infraestructura de desarrollo del país, con lo cual se generen mayores ganancias a las organizaciones $y$, por ende, se retribuyan mayores réditos a los trabajadores.

\section{FIGURA 3}

COSTARRICENSES QUE ESTÁN DE ACUERDO CON LOS CONCEPTOS DEL SECTOR DE LA ECONOMÍA LABORAL

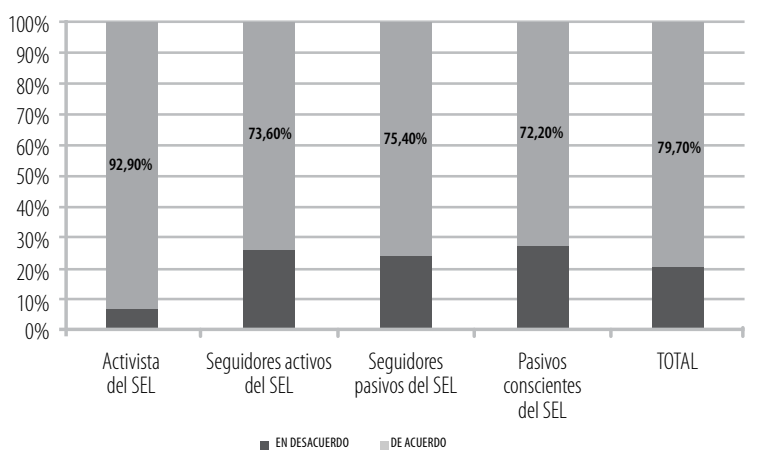

Fuente: Elaboración propia.

\section{Hallazgos más relevantes}

Los hallazgos más relevantes se exponen a continuación:

1. Un $92,1 \%$ de los encuestados considera que el salario devengado no les permite crear un capital.

2. El $78,3 \%$ de las personas considera que los diferentes modelos económicos, tales como el comunismo y el capitalismo, no se preocupan realmente por el bienestar de las personas.

3. Mientras tanto, el $72 \%$ de las personas considera que el Sector de la Economía Laboral (SEL) propone el valor ético del trabajo humano sobre la empresa y el mercado.
4. Los costarricenses participantes de este estudio consideran en un 97\%, que la economía debe ser más humana y solidaria, como lo propone el Sector de la Economía Laboral.

5. Un hallazgo muy importante, representado por el 88,7\% de los costarricenses encuestados, se relaciona con las grandes organizaciones gremiales del país, tales como el Banco Popular y Desarrollo Popular, Junta de Pensiones del Magisterio, Asociación Nacional de Educadores, así como las administradoras de pensiones. Según los informantes, estas instituciones deberían aportar recursos para invertir en la infraestructura del país. Por ejemplo: carreteras, aeropuertos, ferrocarriles, puertos, entre otros proyectos.

6. El $76,4 \%$ de los encuestados considera que la economía debería ser más humana y solidaria, tal y como lo propone el SEL; y ofrecer la ética del trabajo, el empoderamiento de la propiedad productiva en la mayor cantidad de los trabajadores y un mejoramiento en el salario de los trabajadores.

7. Finalmente, un hallazgo muy relevante, no solo por la cantidad de costarricenses, es que el 94,6\% de los encuestados está de acuerdo o muy de acuerdo en que los fondos de las agrupaciones, tales como cooperativismo, solidarismo, pensiones complementarias, fondos complementarios de los trabajadores provenientes de la ley de protección al trabajador, fondos de pensiones, así como los bancos estatales y principalmente el Banco Popular y Desarrollo Comunal, inviertan sus fondos financieros en infraestructura de desarrollo del país por medio del modelo de concesión.

Estos hallazgos son el resultado de la consulta realizada a 3394 personas en los 37 distintos puntos del país, donde la Universidad Estatal a Distancia (UNED) es desde la frontera norte a la frontera sur y de la costa Atlántica a la costa Pa- 
cífica. Con ello, se abarca equitativamente una distribución de la población de Costa Rica.

\section{Corolario}

En esta investigación, se sometió a consulta de los habitantes de Costa Rica la propuesta del Sector de Economía Laboral, la cual, a través del documento, se evidencia que el Gobierno de la República, así como las organizaciones cooperativas, asociaciones solidaristas, juntas de pensiones, Banco Popular y Desarrollo Comunal, Caja de Ande, entre otras, tienen gran interés en definir el Sector de Economía Laboral. También, la academia, tanto en tesis de grado en la Universidad de Costa Rica (UCR) como en la Universidad Nacional (UNA) con publicaciones de libros y artículos sobre el SEL; y tomando en cuenta un libro publicado, un curso de carrera y las investigaciones en la Universidad Estatal a Distancia (UNED), se evidencia que existe un consenso. Tal y como lo expone Malo (1991), en su artículo "Les association sausein de l'économie sociale", en el cual afirma que una propuesta de esta envergadura se da siempre y cuando haya un acuerdo de reconocimiento entre el Estado, la comunidad universitaria y las organizaciones que las conforman. En esta investigación se consultó a la población de Costa Rica acerca de su aceptación y percepción sobre el SEL. A partir de ello, se aprecia cómo el 79,7\% está de acuerdo con todas las propuestas planteadas por el SEL (como se puede apreciar en la ilustración 4) sumado a esto, el grupo de personas identificadas como activistas del SEL, están de acuerdo en un $92,9 \%$.

Se infiere, por tanto, la existencia de una aceptación no tripartita, sino más bien la aprobación cuadripartita, de un cuarto elemento agregado en esta investigación a la propuesta de Malo: el pueblo de Costa Rica. Para aceptar un sector o un tercer sector, como sucedió en el caso de la economía social, con la economía solidaria, se necesita el acuerdo de reconocimiento entre el
Estado, la comunidad universitaria y las organizaciones que conforman el sector.

\section{Conclusión}

En esta investigación se utilizaron herramientas cotidianas en el quehacer académico, sin embargo, pocas veces se conjuntan, por ejemplo: tomar en cuenta a los estudiantes (parte del aprendizaje al realizar una encuesta), la plataforma Moodle, videoconferencias grabadas por la UNED sobre la temática; además, se involucraron los centros universitarios de la universidad, 37 en total, y, como consecuencia, académicos de la universidad.

En el estudio se utilizó el análisis de conglomerados, por medio del método Ward, con los ajustes correspondientes para evitar la generación de sesgos al momento de interpretar los resultados; esto, aunado a la alta fiabilidad conseguida, garantiza que los resultados obtenidos son significativamente seguros.

Uno de los objetivos de esta investigación era validar la propuesta del Sector de la Economía Laboral (SEL), la cual se dio con una aceptación del 79,7\% por parte de los habitantes de Costa Rica. Aunado a ello, se apreció la aceptación del Gobierno de la República (evidenciado en los consultores extranjeros solicitados a la OIT y al Estado de (srael), la Academia o las universidades, en este caso tres de cuatro universidades estatales. También, quedó clara la aceptación de las organizaciones involucradas. Así, lo realizado con la economía social en España, con estos tres últimos actores se cumple con la validación del investigador Malo; no obstante, a esta se añade un cuarto pilar: la consulta de la aceptación o no del SEL. Este trabajo da por sentada la validación de la propuesta del SEL. Sumado a ello, los datos de dicha investigación se expusieron ante la academia nacional e internacional, tanto de forma presente en el Paraninfo Daniel Oduber, de la UNED, como en 22 universidades de 12 países de América y Europa. 
Se concluye que las premisas en las cuales se sustenta el SEL, a saber, entre otras, el valor ético del trabajo, la democracia económica, el empoderamiento de la propiedad productiva, la democratización de la democracia, la repartición equitativa de los ingresos, el trabajador coempresario, el mejoramiento de la infraestructura del país, la creación de una clase media más amplia, son aceptados por las personas participantes en este estudio.

Por otra parte, se colige que los costarricenses consideran a los sindicatos entes pasivos ante los problemas de la clase trabajadora de Costa Rica. De igual forma, manifiestan que el salario no permite crear un capital, el cual contribuya a mejorar su nivel de vida y obtener una pensión digna.

Por último, con referencia a los fondos de capitales sociales, entendiéndose estos como los fondos de Junta de Jubilaciones y Pensiones del Magisterio, Banco Popular y Desarrollo Comunal, cooperativas, asociaciones solidaristas, pensiones complementarias, pensiones de la Caja Costarricense de Seguro Social, Caja de Ande, Seguros del Magisterio, los cuales suman más de $\$ 15000$ millones, el pueblo de Costa Rica indica el deseo de invertirlos en infraestructura, como concesiones de carreteras, puertos, aeropuertos, ferrocarriles, los cuales les permitiría tener mayores ingresos y una pensión mejorada.

\section{REFERENCIAS}

Arauz, A., Levchen, R., Lewinsky, A., Morales, F. y Rojas, R. (1978). El Sector de economía laboral. Cuadernos CEDAL, 8.

Chaverri, P. (1943). El derecho social, como necesidad jurídica contemporánea. SURCO, 40, 28-30.

Dávila, A. y Martínez, N. (s.f.).Cultura en organizaciones latinas. México: Siglo Veintiuno.

De la Cruz, V. (1984). Las luchas sociales en Costa Rica. San José: EUCR/ ECR.

Juliá, J. y Meliá, E. (2009). La economía social en tiempos de crisis. Desafíos y contribuciones. Anuario Iberoamericano de la Economía Social. Madrid: FUNDIBES. Número 1-2009, 234.
Li, F. (2009). La democratización de la propiedad productiva y su contribución al empleo de las empresas cooperativas y asociativas: caso Costa Rica. Jornadas sobre Cooperativismo, Asociativas y Economía solidaría. Herramientas y metodología para su desarrollo. Universidad de la República Montevideo, Uruguay.

Li, F. (2009). Cooperativismo y asociativas en la democratización de la propiedad productiva y su contribución al empleo. Jornadas de trabajo sobre Integración y Cooperación en América Latina. Efectos para el mercado de trabajo y la distribución de la renta. Universidad Santiago de Compostela, Galicia, España.

Li, F. (2012). El Modelo Cooperativo Costarricense: Un enfoque organizacional aplicado a la experiencia costarricense. (Tesis doctoral), UNED, San José.

Li, F. (2013). El solidarismo en la Costa Rica del mañana. San José: CONCASOL.

Malo, M. (1991). Les association sausein de l'économie sociale. Montreal: Inter-Action.

Morales, F. (2008). Costa Rica: Democratizando la democracia. San José: EUNED.

Morales, F. (2012). Sector de economía laboral en Costa Rica (SEL). Academia de Centroamérica. VII Jornada anual. Recuperado de www.academiaca.or.cr/documentos/doc_7162.pdf

Morales, F. (2011). Sector de Economía Laboral en Costa Rica. Anuario Iberoamericano de la Economía Social. Madrid: FUNDIBES. № 2-2011,368

Universidad Estatal a Distancia. (s.f.).Sector de la economía laboral. Recuperado de http://audiovisuales.uned.ac.cr/ videoteca/videos/114/economla-laboral_parte-i-2

Universidad Estatal a Distancia. (s.f.).Sector de la economía laboral. Recuperado de http://audiovisuales.uned.ac.cr/ videoteca/videos/115/economia-laboral_parte-2-2

Universidad Estatal a Distancia (s.f.).Sector de la economía laboral. Recuperado de http://audiovisuales.uned.ac.cr/ videoteca/videos/207/vc-1-economla-laboral-parte-2

Universidad Estatal a Distancia (s.f.).Sector de la economía laboral. Recuperado de http://audiovisuales.uned.ac.cr/ videoteca/videos/210/vc-4-economla-laboral

Universidad Estatal a Distancia (s.f.).Sector de la economía laboral. Recuperado de http://audiovisuales.uned.ac.cr/ videoteca/videos/207/vc-1-economla-laboral-parte-2

Recibido: 19 de setiembre de 2013 Aceptado: 20 de noviembre de 2013 